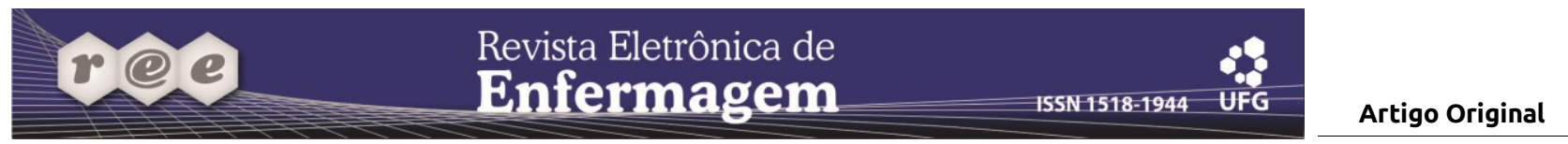

\title{
Prevalência dos fatores de risco para diabetes mellitus de servidores públicos
}

\author{
Prevalence of risk factors for diabetes mellitus among public servants
}

Prevalencia de los factores de riesgo para diabetes mellitus de servidores públicos

Carla Campos Muniz Medeiros ${ }^{1}$, Galdino Glauber Bessa², Alexsandro Silva Coura ${ }^{3}$, Inacia Sátiro Xavier de França ${ }^{4}$, Francisco Stélio de Sousa ${ }^{5}$

\section{RESUMO}

Este estudo objetivou verificar a prevalência dos fatores de risco para diabetes mellitus tipo 2 de servidores públicos, e relacionar os fatores de risco segundo as variáveis, sexo e glicemia. Participaram 65 sujeitos, mediante um questionário, e aferiram-se: pressão arterial, peso corporal, altura, índice de massa corpórea, circunferência abdominal e glicemia capilar de jejum. Utilizaram-se análise de variância e qui-quadrado. Os fatores de risco mais prevalentes foram: índice de massa corpórea alterado $(66,1 \%)$, idade $>45$ anos $(64,6 \%)$, circunferência abdominal alterada (61,5\%) e sedentarismo (61,5\%). Observou-se associação entre a pressão arterial e a glicemia capilar de jejum alterada $(p=0,01)$. Os homens apresentaram maior prevalência de glicemia capilar de jejum alterada $(p<0,035)$ e índice de massa corpórea alterado $(p<0,007)$. Conclui-se que os fatores de risco para o diabetes mellitus tipo 2 possuem elevada prevalência na população estudada, refletindo um estilo de vida inadequado para a prevenção desta doença.

Descritores: Cuidados de Enfermagem; Diabetes Mellitus; Fatores de Risco.

\section{ABSTRACT}

The objective was to verify the prevalence of risk factors for type 2 diabetes mellitus among public servants, and relate risk factors to gender and blood glucose variables. The participants were 65 public servants who answered a questionnaire. The following measures were obtained: blood pressure, body weight, height, body mass index, abdominal circumference, and fasting capillary blood glucose. Analysis of variance and the chi-square test were used. The most prevalent risk factors identified were: abnormal body mass index (66.1\%), age>45 years (64.6\%), abnormal abdominal circumference (61.5\%) and sedentary life style (61.5\%). An association was observed between blood pressure and altered fasting capillary blood glucose $(p=0.01)$. Men showed a higher prevalence of altered capillary blood glucose $(p<0.035)$ and altered body mass index $(p<0.007)$. In conclusion, the risk factors for type 2 diabetes mellitus are highly prevalent in the studied population, revealing an inadequate life style for the prevention of this disease.

Descriptors: Nursing Care; Diabetes Mellitus; Risk Factors.

\section{RESUMEN}

Se objetivó verificar la prevalencia de factores de riesgo para diabetes mellitus tipo 2 de servidores públicos y relacionar tales factores según las variables sexo y glucemia. Participaron 65 sujetos, mediante un cuestionario, y se compararon: presión arterial, peso corporal, altura, índice de masa corporal, circunferencia abdominal y glucemia sanguínea en ayunas. Se utilizaron análisis de varianza y Chi-cuadrado. Los factores de riesgo más prevalentes fueron: índice de masa corporal alterado (66,6\%), edad>45 años (64,6\%), circunferencia abdominal alterada (61,5\%) y sedentarismo (61,5\%). Se observó asociación entre presión arterial y glucemia sanguínea en ayunas alterada $(p=0,01)$. Los hombres expresaron mayor prevalencia de glucemia sanguínea en ayunas alterada $(p<0,035)$ e índice de masa corporal alterado $(p<0,007)$. Se concluye en que los factores de riesgo para diabetes mellitus tipo 2 poseen elevada prevalencia en la población estudiada, reflejando un estilo de vida inadecuado para prevenir la enfermedad.

Descriptores: Atención de Enfermería; Diabetes Mellitus; Factores de Riesgo.

\footnotetext{
${ }^{1}$ Médica. Professora Doutora da Universidade Estadual da Paraíba (UEPB). Campina Grande-PB. E-mail: carlamunizmedeiros@hotmail.com.

${ }^{2}$ Enfermeiro. Campina Grande, PB, Brasil. E-mail: bessa@uepb.edu.br.

${ }^{3}$ Enfermeiro, Mestre em Saúde Pública. Discente do Programa de Pós-Graduação em Enfermagem da Universidade Federal do Rio Grande do Norte. Natal, RN, Brasil. E-mail: alex@uepb.edu.br.

${ }^{4}$ Enfermeira, Doutora em Enfermagem. Docente do Departamento de Enfermagem da UEPB. Campina Grande, PB, Brasil. E-mail: isxf@oi.com.br.

${ }^{5}$ Enfermeiro, Doutor em Enfermagem. Professor Doutor da UEPB. Campina Grande, PB, Brasil. E-mail: stelio uepb@yahoo.com.br.
} 


\section{INTRODUÇÃO}

O Diabetes Mellitus tipo 2 (DM2), permanece um grave problema de saúde pública afetando, aproximadamente, 173 milhões de indivíduos em todo o mundo. Esse agravo se caracteriza como uma doença crônica, devido à falta de insulina ou à incapacidade da insulina para exercer adequadamente seus efeitos, sendo mais comum em indivíduos com excesso de peso. Estima-se que, para o ano de 2030, em todo o mundo, existirá uma população de 300 milhões de pessoas sofrendo de diabetes, e que essa doença seja uma das principais causas de morte ${ }^{(1)}$.

Consta na literatura(2) que, atualmente, existem no mundo mais de 1,1 bilhão de adultos com sobrepeso e 312 milhões que estão obesos. Nas comunidades hispânicas dos Estados Unidos, dos imigrantes árabes, chineses e indianos que se submeteram à ocidentalização e à urbanização, a prevalência de diabetes está na faixa de 14 a 20\%. E estima-se que a população asiática tenha alto risco para o DM devido à obesidade abdominal que pode levar à síndrome metabólica e tolerância à glicose diminuída. Outrossim, no noroeste de Adelaide, sul da Austrália, realizou-se estudo $^{(3)}$ com 263 adultos com idade de 18 anos ou mais, para identificação de DM autorreferido. Obteveram-se fatores associados ao DM para homens e mulheres com 40 anos e mais: renda familiar bruta anual baixa, obesidade e história familiar de diabetes.

Pesquisadores brasileiros ${ }^{(4)}$ investigaram a razão de prevalência do DM nas diferentes regiões geográficas do país, no período 2002-2007, detectando-se alta prevalência de DM, com destaque para o Distrito Federal e o Estado do Maranhão. Os pesquisadores fundamentaram o estudo em relato de investigações realizadas nos Estados Unidos, Canadá, Grécia, Reino Unido e Austrália, dentre outros, nos quais se obteve aumento progressivo do DM no mesmo interstício desse estudo brasileiro. Em 2006, em outra pesquisa(5) realizada em 27 cidades de diversas regiões brasileiras, identificou-se que a prevalência do diabetes foi mais acentuada nos sujeitos na faixa etária dos 45-54 anos, principalmente naquelas pessoas pré-obesas e obesas. $E$ estimou-se a existência de 6.317 .621 casos diagnosticados de diabetes, sendo 2.573 .413 homens e 3.744.208 mulheres.
Na Paraíba, em estudo com 22 pacientes cadastrados no HIPERDIA, a maioria era de mulheres com mais de 40 anos, casadas, sem escolaridade, aposentadas e com renda familiar de um salário mínimo. Verificou-se que $100 \%$ da amostra usavam, apenas, tratamento medicamentoso para controle metabólico, e estavam expostas a fatores de riscos sistêmicos e locais, como hipertensão arterial e o uso de sapatos abertos, além da adesão parcial ao tratamento e baixo conhecimento sobre as complicações crônicas'(6). Em outro estudo paraibano, investigaram-se 510 idosos com DM. Destes, 54,5\% estavam descompensados metabolicamente, $62,7 \%$ usavam hipoglicemiante, e $97,8 \%$, dieta prescrita. Identificou-se prejuízo no tratamento devido ao baixo índice de retornos $(11,9 \%)$ no período de estudo(7). Em Campina Grande, Estado da Paraíba, em estudo com 103 usuários, sendo 21,4\% homens e $78,6 \%$ mulheres, cadastrados em uma Unidade Básica de Saúde da Família, identificou-se que 5,8\% dos sujeitos apresentavam risco aumentado para desenvolver diabetes ${ }^{(8)}$.

Os elevados índices epidemiológicos e as complicações decorrentes do DM, tais como a neuropatia periférica, as ulcerações dos pés, a amputação do membro afetado(9) - que podem levar à invalidez, os enfartes do miocárdio, o derrame cerebral, a impotência, a nefropatia, as retinopatias que levam à cegueira, as frequentes hospitalizações, o alto custo do tratamento e a perda de anos de vida por morte prematura - são exemplos do impacto negativo que o DM2 traz para a sociedade ${ }^{(10)}$. Dentre os fatores de risco (FR) para o surgimento do DM2 destacam-se: antecedentes familiares, o consumo de dietas hipercalóricas, o sobrepeso, o sedentarismo, as mudanças socioculturais introduzidas pela industrialização e o aumento na expectativa de vida da população(2).

Adultos jovens, envolvidos com a busca de estabilidade profissional, tornam-se vulneráveis ao consumismo contemporâneo ${ }^{(11)}$, aderindo à alimentação rápida e prática, ao sedentarismo e ao sobrepeso influenciados pela evolução tecnológica que minimiza o esforço físico nas atividades cotidianas, contribuindo com a predisposição dos indivíduos ao $\mathrm{DM}^{(12)}$. No caso deste estudo, os técnicos administrativos trabalhavam nos turnos diurnos, com duas horas de intervalo para o 
almoço. O campus universitário, cenário da pesquisa, situa-se em um bairro periférico, dificultando o deslocamento desses profissionais até suas residências no horário das refeições. Assim, consomem-se sanduíches, massas, refrigerantes e doces disponíveis na praça de alimentação do campus. A Universidade Estadual da Paraíba (UEPB) sedia uma Clínica de Enfermagem em que se desenvolvem atividades de educação em saúde e a prevenção e controle da hipertensão e do DM por meio de consulta de enfermagem. Entretanto, há resistência dos técnicos administrativos à participação nas atividades educativas. Dada a subutilização das ações educativas/preventivas desenvolvidas na Clínica de Enfermagem e/ou subestimação das práticas de autocuidado, acredita-se existir elevada prevalência dos FRs para DM2 entre os servidores técnicos administrativos da UEPB.

Justifica-se o estudo devido ao aumento progressivo dos casos de DM2 e ao impacto social dessa patologia. Na busca de artigos realizada na Biblioteca Virtual em Saúde (BVS), utilizando-se os índices e fontes disponíveis, foram detectados 2.743 artigos referentes ao cruzamento das palavras "diabetes mellitus e prevalência", mas obteve-se apenas um artigo em que se avaliou os FRs em servidores públicos estaduais(13). Entende-se que os seus resultados possibilitam a realização de outros estudos na mesma temática em contextos mais amplos, o debate sobre os FRs para DM em trabalhadores do serviço público, e o planejamento, por parte dos gestores, de estratégias e ações administrativas que reduzam a vulnerabilidade dos trabalhadores a comportamentos de risco para doenças crônicas não transmissíveis. E que o enfermeiro tem o papel social de educar os usuários do sistema de saúde visando à prevenção, ao controle de doenças e à promoção da saúde.

No caso específico do enfermeiro, os resultados deste estudo podem contribuir para o compartilhamento de informações entre poder público, profissionais de saúde e pacientes, visando à promoção da qualidade da assistência em saúde ${ }^{(14)}$, com a oferta de intervenções clínicas e educativas baseadas em evidências científicas que contribuam para a elaboração de protocolos que subsidiem o planejamento da assistência, o alcance de controle metabólico e a manutenção da saúde ocupacional. Norteado por esse pressuposto, o estudo objetivou verificar a prevalência dos fatores de risco para DM2 de servidores públicos, e relacionar os fatores de risco segundo as variáveis sexo e glicemia.

\section{MÉTODOS}

Estudo descritivo, transversal e quantitativo, realizado entre os meses de agosto e outubro de 2008, na Clínica de Enfermagem do Centro de Ciências Biológicas e da Saúde (CCBS), da UEPB, em Campina Grande/PB.

A população alvo foram os 98 servidores técnicoadministrativos do CCBS, lotados em um destes departamentos: Enfermagem, Educação Física, Odontologia, Fisioterapia, Farmácia, Biologia e Psicologia, além daqueles com atuação na diretoria do Centro ou na Central Telefônica.

A amostra foi calculada aplicando-se a fórmula: $\mathrm{n}=$ N. $Z^{2} \cdot P(1-P) /(N-1) \cdot e^{2}+Z^{2} \cdot P(1-P)^{(15)}$, considerando uma prevalência de $15,02 \%{ }^{(16)}$. Obteve-se um $n$ amostral de 66 , o qual foi corrigido em $5 \%$ para compensar eventuais perdas ou problemas operacionais da pesquisa, sendo necessário um n de 69 sujeitos. Os critérios de inclusão foram: ser servidor técnico-administrativo lotado no CCBS e concordar com a participação. E os critérios de exclusão: estar afastado das funções trabalhistas, referir diagnóstico prévio de diabetes, usar corticóides ou outra medicação que interfira no metabolismo glicídico.

Foram excluídos 33 funcionários porque sete se recusaram participar, sete foram transferidos para outros centros, quatro estavam de licença ou férias, dois declararam estar usando corticóide, quatro estavam se aposentando e nove referiram DM2. Portanto, a amostra definitiva foi composta por 65 sujeitos. Salienta-se que, mesmo com a participação de quatro sujeitos a menos do $n$ calculado, essa amostra é representativa da população, pois o erro foi inferior a $5 \%$, valor considerado como permitido para o cálculo do tamanho de amostras(15).

Utilizou-se um questionário, composto por 21 questões fechadas referentes aos dados sociodemográficos (idade, sexo, cor, renda e escolaridade) e fatores de risco. E a mensuração da altura, do peso corporal, da pressão arterial (PA), da circunferência abdominal (CA), da glicemia capilar de jejum (GCJ) e do índice de massa corporal (IMC). Com relação à classificação dos fatores de risco, 
consideraram-se: I. Fatores de risco não modificáveis idade > 45 anos e antecedente familiar; e II. Fatores de risco modificáveis - obesidade, circunferência abdominal alterada, índice de massa corporal alterado, sedentarismo, hipertensão arterial sistêmica, pressão arterial alterada, glicemia capilar de jejum alterada e cardiopatias.

A coleta dos dados ocorreu em duas etapas. 1) Procedeu-se a uma visita à direção do CCBS, à Central Telefônica e aos departamentos para conhecer a rotina de cada setor e convidar cada servidor a participar da pesquisa. Para os que aceitaram, foram agendados dia e hora para comparecimento individual à Clínica de Enfermagem para preenchimento do questionário. 2) Aplicou-se o questionário e realizou-se a mensuração do peso e altura, da PA, da CA, da GCJ e do IMC.

Verificou-se o peso corporal por meio de uma balança antropométrica, com precisão de 0,05 Kg, da marca Filizola. A mensuração da altura foi obtida usandose o estadiômetro correspondente à balança antropométrica utilizada, com precisão de $0,5 \mathrm{~cm}$, estando o sujeito em posição ortostática e com os pés juntos ${ }^{(17)}$.

A PA foi aferida por método auscultatório em dois momentos (fases I e V dos ruídos de Korotkoff), utilizando-se um esfingmomanômetro após 10 minutos com o sujeito na posição sentada. Foi considerada a média das três verificações da PA, e PA elevada aquela $\geq$ $140 \times 90 \mathrm{mmHg}^{(18)}$.

Рага a verificação da CA, empregou-se fita métrica com precisão de $0,1 \mathrm{~cm}$. A efetuação da medida ocorreu com os indivíduos na posição ortostática. Para identificação do valor, foi verificada a circunferência no nível do ponto médio entre a última costela e a crista ilíaca, no final do movimento expiratório. Foram considerados como CA alterada os valores $\geq 94 \mathrm{~cm}$ para homens e $\geq 80 \mathrm{~cm}$ para mulheres ${ }^{(18)}$.

O teste glicêmico capilar se realizou com o sujeito em jejum de, no mínimo, oito horas, mediante punção da polpa digital utilizando uma lanceta e coleta de uma gota de sangue que foi adicionada a fita pré-colocada no glicosímetro da Advantage Perfomace, Roche ${ }^{\circledR}$. Consideraram-se como GCJ alterada os valores $\geq 100$ $\mathrm{mg} / \mathrm{dL}^{(15)}$.

Para calcular o IMC aplicaram-se os dados do peso corporal e da altura na fórmula: IMC = Peso $(\mathrm{kg}) /$ Altura $^{2}(\mathrm{~m})$. Consideraram-se como IMC alterado (sobrepeso/obesidade) os valores acima de 25 $\left(\mathrm{kg} / \mathrm{m}^{2}\right)^{(15)}$.

Os dados coletados foram implantados e analisados no software Statistical Package for the Social Sciences (SPSS) versão 17.0. Utilizou-se a estatística descritiva para analisar os dados, sendo calculadas as frequências absolutas e relativas, as médias e o desvio padrão, para descrição e caracterização da amostra estudada. A comparação de médias da glicemia capilar foi feita por meio do teste $t$ de Student. Para comparar as proporções da prevalência dos FR foi utilizado o teste do qui-quadrado. As análises estatísticas se realizaram considerando intervalo de confiança de $95 \%$.

O projeto foi aprovado no Comitê de Ética da UEPB, sob o número do processo 0165.0.133.000-08. Em concordância com a Resolução 196/96 do Conselho Nacional de Saúde, os sujeitos foram esclarecidos acerca dos objetivos do estudo, leram e assinaram o Termo de Consentimento Livre e Esclarecido, e tiveram assegurados a privacidade, confidencialidade, anonimato e a liberdade de não participar ou desistir do estudo.

\section{RESULTADOS}

\section{Perfil sociodemográfico}

Na Tabela 1 é apresentado o perfil sociodemográfico dos servidores técnico-administrativos do CCBS/UEPB. Entre os sujeitos que participaram do estudo ocorreu paridade aproximada entre os sexos, tendo o masculino apenas um representante a mais. A média de idade foi de 48 anos, com predomínio das pessoas que tinham mais de 41 anos $(84,6 \% ; n=55)$. 
Tabela 1: Perfil sociodemográfico dos servidores técnico-administrativos do CCBS da UEPB ( $n=65)$. Campina Grande, PB, 2008.

\begin{tabular}{|c|c|c|}
\hline Variáveis & $\mathbf{n}$ & $\%$ \\
\hline \multicolumn{3}{|l|}{ Sexo } \\
\hline Masculino & 33 & 50,8 \\
\hline Feminino & 32 & 49,2 \\
\hline \multicolumn{3}{|l|}{ Faixa Etária } \\
\hline $20-30$ anos & 6 & 9,2 \\
\hline $31-40$ anos & 4 & 6,2 \\
\hline $41-50$ anos & 27 & 41,5 \\
\hline Mais de 50 anos & 28 & 43,1 \\
\hline \multicolumn{3}{|l|}{ Cor } \\
\hline Branco & 35 & 53,8 \\
\hline Não branco & 30 & 46,2 \\
\hline \multicolumn{3}{|l|}{ Situação Conjugal } \\
\hline Sem companheiro & 38 & 58,5 \\
\hline Com companheiro & 27 & 41,5 \\
\hline \multicolumn{3}{|l|}{ Escolaridade } \\
\hline Ensino médio incompleto & 34 & 52,3 \\
\hline Ensino médio completo & 14 & 21,5 \\
\hline Superior incompleto & 8 & 12,3 \\
\hline Superior completo & 4 & 6,2 \\
\hline Pós-Graduação & 5 & 7,7 \\
\hline \multicolumn{3}{|l|}{ Outra Atividade Remunerada } \\
\hline Sim & 11 & 16,9 \\
\hline Não & 54 & 83,1 \\
\hline \multicolumn{3}{|l|}{ Renda Familiar* } \\
\hline Um a três salários mínimos & 38 & 58,5 \\
\hline Quatro a seis salários mínimos & 18 & 27,7 \\
\hline Mais de seis salários mínimos & 9 & 13,8 \\
\hline
\end{tabular}

*Salário mínimo = R\$ 420,00

\section{Fatores de risco}

A Tabela 2 apresenta a prevalência dos FR modificáveis e não modificáveis identificados na amostra, bem como os valores de fatores acumulados entre os sujeitos. Entre os fatores modificáveis as maiores prevalências foram do sedentarismo, da CA e IMC alterados. Dos sujeitos que afirmaram realizar atividade física ( $n=25)$, apenas $40 \%$ disseram realizá-la diariamente, sendo a caminhada a atividade mais praticada (40\%). Já a média da glicemia capilar de jejum dos sujeitos foi de $98,55 \mathrm{mg} / \mathrm{dL}$. O desvio padrão foi de 44,55 , observando-se uma prevalência de $20 \%$ de glicemia alterada. 
Tabela 2: Prevalência de fatores de risco para diabetes mellitus tipo 2 em servidores técnico-administrativos do CCBS da UEPB e número acumulado de fatores (n=65). Campina Grande, PB, 2008.

\begin{tabular}{|c|c|c|}
\hline Fatores de risco & $\mathbf{n}$ & $\%$ \\
\hline \multicolumn{3}{|l|}{ Não-modificáveis } \\
\hline Idade $>45$ anos & 42 & 64,6 \\
\hline Antecedente familiar & 34 & 52,3 \\
\hline \multicolumn{3}{|l|}{ Modificáveis } \\
\hline Obesidade $^{*}$ & 06 & 9,2 \\
\hline Circunferência abdominal alterada & 40 & 61,5 \\
\hline Índice de massa corporal alterado & 43 & 66,1 \\
\hline Sedentarismo* & 40 & 61,5 \\
\hline Hipertensão arterial sistêmica* & 20 & 30,8 \\
\hline Pressão arterial alterada & 20 & 30,8 \\
\hline Glicemia capilar de jejum alterada & 13 & 20,0 \\
\hline Cardiopatias* & 05 & 7,7 \\
\hline \multicolumn{3}{|c|}{ Número de fatores de risco não-modificáveis } \\
\hline Nenhum fator de risco & 12 & 18,5 \\
\hline Um fator de risco & 32 & 49,2 \\
\hline Dois fatores de risco & 21 & 32,3 \\
\hline \multicolumn{3}{|c|}{ Número de fatores de risco modificáveis } \\
\hline Nenhum fator de risco & 07 & 10,8 \\
\hline Um ou dois fatores & 27 & 41,5 \\
\hline Três a cinco fatores & 31 & 47,7 \\
\hline \multicolumn{3}{|l|}{ Número global de fatores de risco } \\
\hline Nenhum fator de risco & - & - \\
\hline Um a dois fatores de risco & 17 & 26,1 \\
\hline Três fatores de risco & 18 & 27,7 \\
\hline Quatro ou mais fatores de risco & 30 & 46,2 \\
\hline
\end{tabular}

${ }^{*}$ Condições autorreferidas.

\section{Associações entre os fatores de risco}

A Tabela 3 mostra que ocorreu equilíbrio na frequência de sedentarismo $(n=25)$ entre os sexos, cada um apresentando 20 sujeitos que não praticavam atividade física, fazendo com que não houvesse relevância estatística na amostra que estabelecesse relação entre sedentarismo e sexo $(p>0,05)$. Os fatores de risco HAS, IMC e GCJ foram mais prevalentes no sexo masculino, com destaque para os dois últimos, que se mostraram estatisticamente relacionados com o sexo $p<0,007$ e $p<0,035$, respectivamente. O sexo feminino teve predominância em apenas um fator de risco, que foi a CA, no entanto não apresentou significância estatística $(p>0,05)$. 
Tabela 3: Distribuição dos servidores técnico-administrativos do CCBS da UEPB, conforme a relação entre os fatores de risco para DM2 e o sexo ( $n=65)$. Campina Grande, PB, 2008.

\begin{tabular}{|c|c|c|c|c|c|}
\hline \multirow[t]{3}{*}{ Fatores de risco } & \multicolumn{5}{|c|}{ Sexo $(n=65)$} \\
\hline & \multicolumn{2}{|c|}{ Masculino ( $n=33)$} & \multicolumn{2}{|c|}{ Feminino ( $n=32)$} & \multirow[t]{2}{*}{ p-valor } \\
\hline & $n$ & $\%$ & $\mathbf{N}$ & $\%$ & \\
\hline \multicolumn{6}{|l|}{ Idade } \\
\hline$\leq 45$ anos $(n=23)$ & 15 & 45,5 & 08 & 25 & 0,085 \\
\hline$>45$ anos $(n=42)$ & 18 & 54,5 & 24 & 75 & \\
\hline \multicolumn{6}{|l|}{ Antecedentes familiares } \\
\hline Não (n=34) & 14 & 42,4 & 20 & 62,5 & 0,105 \\
\hline $\operatorname{Sim}(n=31)$ & 19 & 57,6 & 12 & 37,5 & \\
\hline \multicolumn{6}{|l|}{ Sedentarismo } \\
\hline Não $(n=40)$ & 20 & 60,6 & 20 & 62,5 & 0.875 \\
\hline $\operatorname{Sim}(n=25)$ & 13 & 39,4 & 12 & 37,5 & \\
\hline \multicolumn{6}{|l|}{ IMC } \\
\hline Normal ( $n=22)$ & 06 & 18,2 & 16 & 50 & 0,007 \\
\hline Sobrepeso/obesidade $(n=43)$ & 27 & 81,8 & 16 & 50 & \\
\hline \multicolumn{6}{|l|}{ Pressão Arterial } \\
\hline$<140 \times 90 \mathrm{mmHg}(\mathrm{n}=45)$ & 22 & 66,7 & 23 & 71,9 & 0,649 \\
\hline$\geq 140 \times 90$ mmHg $(n=20)$ & 11 & 33,3 & 09 & 28,1 & \\
\hline \multicolumn{6}{|l|}{ Circunferência Abdominal } \\
\hline Normal $(n=25)$ & 14 & 42,4 & 11 & 34,4 & 0,505 \\
\hline Alterada $(n=40)$ & 19 & 57,6 & 21 & 65,6 & \\
\hline \multicolumn{6}{|l|}{ Glicemia de Jejum } \\
\hline Normal $(n=52)$ & 23 & 69,7 & 29 & 90,6 & 0,035 \\
\hline Alterada $(n=13)$ & 10 & 30,3 & 03 & 9,4 & \\
\hline
\end{tabular}

Na Tabela 4, verifica-se que a única variável que apresentou relação estatisticamente significativa com a GCJ alterada foi a pressão arterial $(p=0,01)$. 
Tabela 4: Distribuição dos servidores técnico-administrativos do CCBS da UEPB, conforme a relação entre glicemia capilar de jejum e sua associação com os fatores de risco para DM2 (n=65). Campina Grande, PB, 2008.

\begin{tabular}{|c|c|c|c|c|c|}
\hline \multirow{3}{*}{ Fatores de risco } & \multicolumn{4}{|c|}{ Glicemia (n=65) } & \multirow{3}{*}{ p-valor } \\
\hline & \multicolumn{2}{|c|}{ Alterada $(n=13)$} & \multicolumn{2}{|c|}{ Normal $(n=52)$} & \\
\hline & $\mathbf{n}$ & $\%$ & $\mathbf{n}$ & $\%$ & \\
\hline \multicolumn{6}{|l|}{ Idade } \\
\hline$\leq 45$ anos $(n=23)$ & 03 & 23,1 & 20 & 38,5 & 0,29 \\
\hline$>45$ anos $(n=42)$ & 10 & 76,9 & 32 & 61,5 & \\
\hline \multicolumn{6}{|l|}{ Antecedentes familiares } \\
\hline Não $(n=34)$ & 05 & 38,5 & 29 & 55,8 & 0,26 \\
\hline $\operatorname{Sim}(n=31)$ & 08 & 61,5 & 23 & 44,2 & \\
\hline \multicolumn{6}{|l|}{ Sedentarismo } \\
\hline Não $(n=40)$ & 06 & 46,2 & 19 & 36,5 & 0,52 \\
\hline $\operatorname{Sim}(n=25)$ & 07 & 53,8 & 33 & 63,5 & \\
\hline \multicolumn{6}{|l|}{ IMC } \\
\hline Normal (n=22) & 02 & 15,4 & 20 & 38,5 & 0,11 \\
\hline Sobrepeso/obesidade ( $n=43$ ) & 11 & 84,6 & 32 & 61,5 & \\
\hline \multicolumn{6}{|l|}{ Pressão Arterial } \\
\hline$<140 \times 90 \mathrm{mmHg}(\mathrm{n}=45)$ & 04 & 30,8 & 41 & 78,8 & 0,01 \\
\hline$\geq 140 \times 90 \mathrm{mmHg}(n=20)$ & 09 & 69,2 & 11 & 21,2 & \\
\hline \multicolumn{6}{|l|}{ Circunferência Abdominal } \\
\hline Normal $(n=25)$ & 02 & 15,4 & 23 & 44,2 & 0,56 \\
\hline Alterada $(n=40)$ & 11 & 84,6 & 29 & 55,8 & \\
\hline
\end{tabular}

\section{DISCUSSÃO}

A caracterização da amostra revelou praticamente a mesma proporção entre homens e mulheres, diferindo do resultado de outro estudo em que, dos 198 indivíduos estudados, 68 (34,4\%) eram homens, e 130 $(65,6 \%)$, mulheres ${ }^{(20)}$, e também dos resultados do inquérito de morbidade autorreferida realizado nas capitais brasileiras e no Distrito Federal(6), em que as mulheres foram maioria. É preocupante a verificação de escolaridades e renda relativamente baixas na amostra, pois as características socioeconômicas de uma população podem ser relevantes na prevenção e no controle do $\mathrm{DM}^{(3)}$.

Os FR apresentados pela amostra confirmam dados de outros estudos no concernente a: idade - autores relatam cerca de 113 milhões de pessoas com DM que se incluem na faixa etária dos sujeitos do estudo atual, o que representa $46 \%$ das pessoas com a doença ${ }^{(10)}$; o predomínio de DM2 em indivíduos não brancos contradiz resultado de estudo em que a glicemia de jejum não diferiu entre pacientes negros e brancos ${ }^{(21)}$.

No concernente aos FR não modificáveis verificouse, além de frequência elevada de sujeitos com idade avançada, alta prevalência de antecedentes familiares para DM2. Dado importante, pois familiares de primeiro grau de pessoas com DM2 apresentam de duas a seis vezes mais chance de desenvolver diabetes do que controles sem história familiar. Outrossim, o caráter assintomático do DM propicia o diagnóstico tardio e o desencadear de complicações $^{(7)}$. No tocante aos FR modificáveis, merece destaque o fato de quase metade da amostra ter autorreferido de três a cinco FR, quais sejam: obesidade, HAS, sedentarismo e cardiopatias, confirmando resultados de outro estudo em que a história familiar de diabetes e hipertensão foi identificada em $45 \%$ da amostra. A prevalência de sedentarismo foi igual a $75 \%$; de sobrepeso, igual a $15 \%$; e de obesidade (IMC> $\left.25 \mathrm{~kg} / \mathrm{m}^{2}\right)$, igual a $5 \%{ }^{(22)}$.

A associação do DM2 com a HAS é encontrada na prática clínica, sugerindo que a HAS pode aumentar a chance de o indivíduo desenvolver diabetes; tal como demonstrado em relato de estudo no qual se estimou que, no Brasil, há 6.317.621 adultos que referem ter diabetes e, destes, 25.690.145 referem ter hipertensão(5). No presente estudo a única variável que apresentou relação estatística significante com a GCJ alterada foi a pressão arterial $(p=0,01)$. Essa mesma associação foi encontrada em um estudo realizado no 
Hospital das Clínicas de Porto Alegre, que objetivou identificar a prevalência dos FR cardiovasculares em indivíduos com DM ou GCJ alterada. Esta associação é uma das complicações no tratamento da DM, pois existem evidências indicando que o controle pressórico é mais efetivo na redução de complicações macrovasculares do DM do que o controle glicêmico(23).

O desvio padrão elevado da GCJ indica que alguns sujeitos estavam com a taxa glicêmica muito elevada. Tal inferência foi confirmada ao constatar-se que, dos 13 sujeitos com GCJ elevada, quatro apresentaram valores muito acima de $126 \mathrm{mg} / \mathrm{dL}$ (critério para diagnóstico do diabetes), sendo necessária apenas a confirmação por teste laboratorial para fechar diagnóstico de diabetes.

Grande parcela da amostra deste estudo referiu alguma das morbidades que tanto aumentam o risco de DM2, como na sua presença agravam a doença. Esse fato conduz à ótica de risco aumentado para os indivíduos que referiram diagnóstico dessas morbidades, principalmente, quando associadas a algum outro FR para o DM2. Os fatores de risco mais prevalentes - CA alterada, IMC alterado e sedentarismo - são passíveis de intervenções com mudanças no estilo de vida e realização de atividade física associada a uma alimentação equilibrada. Entre as pessoas que praticavam atividades físicas, a caminhada foi a mais citada. Todavia, tal exercício é considerado leve e, dependendo do tempo de sua realização, pode não interferir significativamente na prevenção do DM2, pois os benefícios acontecem com atividades de intensidade vigorosa ou moderada(24).

No tocante à relação entre CA e IMC, mesmo que a existência de uma variável não indique, necessariamente, a existência da outra, elas correlacionam-se e são fortes FR para o DM2, aumentando o risco da doença quando as duas variáveis coexistem no indivíduo(1). Um dado preocupante é que $87,2 \%$ da população total do estudo apresentava algum tipo de sobrepeso/obesidade na forma de IMC alterado ou CA alterada. Não ocorreu associação estatísticamente significativa entre as variáveis sedentarismo e sexo masculino ou feminino.

As diferenças estatisticamente significantes identificadas entre homens e mulheres no tocante à prevalência de GCJ e IMC alterados, com maior frequência no sexo masculino, divergem de um estudo desenvolvido em 27 cidades brasileiras, que identificou maior prevalência de diabetes autorreferida no sexo feminino 6,0\% (IC 95\%: 5,7;6,2) do que no sexo masculino 4,4\% (IC 95\%: 4,2;4,7)(5). Outra pesquisa, realizada em um município do interior de Pernambuco, também diverge nesse sentido, sendo a prevalência de DM nos homens de $8,8 \%$ e nas mulheres de $16,2 \%$.

As limitações do estudo são da seguinte ordem: utilização de amostra reduzida, retratando uma realidade local, em detrimento de uma possível generalização dos resultados; considerar algumas condições de saúde autorreferidas; e impossibilidade de mensuração do colesterol HDL e/ou triglicérideos, sendo os mesmos importantes marcadores de DM2. Além disso, os estudos transversais dificultam determinar a direcionalidade das associações devido ao risco de viés da causalidade reversa, uma vez que fatores de risco, de proteção e desfechos são verificados concomitantemente.

\section{Implicações do estudo para a enfermagem}

Por mais que os estudos científicos apresentem tratamentos comprovados e inovadores para o DM, por mais efetivas que sejam as intervenções comportamentais implementadas nos estudos prospectivos e controlados, estes não surtirão efeito se o paciente não seguir à risca o tratamento e não adotar, definitivamente e ao longo do tempo, as mudanças comportamentais( $^{(9)}$. Assim, cabe aos enfermeiros a utilização de ferramentas para a implantação de protocolos para atendimento ao portador de DM, como a consulta de enfermagem com foco no autocuidado ${ }^{(25)}$. E também capacitar o paciente e os familiares para o manejo do DM, informando-os sobre a vulnerabilidade ao DM, como prevenir esse agravo, motivando-os para o autocuidado, reforçando os comportamentos proativos, decidindo junto com eles estratégias e ações preventivas do DM e responsabilizando-os pela adoção de condutas mais eficazes para a prevenção ou o controle do DM, de modo a prevenir a ocorrência de uma série de doenças crônicas no futuro.

No caso específico dos sujeitos deste estudo, sugere-se o incremento de um projeto de extensão em parceria com outros departamentos da instituição, a exemplo dos departamentos de Educação Física, Fisioterapia, Odontologia e de Psicologia, compondo uma equipe interdisciplinar que estimule e oriente esses 
indivíduos a valorizar a aquisição de estilo de vida saudável e para que se comprometam com a prática diária de autocuidado.

\section{CONSIDERAÇÕES FINAIS}

Conclui-se que os FR para o DM2 possuem elevada prevalência na população estudada, refletindo um estilo de vida inadequado para a prevenção de tal patologia. Verificou-se também associação entre PA e GCJ, bem como que os homens apresentaram maior prevalência de GCJ e IMC alterados. Esses resultados apontam para a necessidade de se planejar e implementar ações de

\section{REFERÊNCIAS}

1. Diretrizes da Sociedade Brasileira de Diabetes 2009. Sociedade Brasileira de Diabetes. 3a ed. Itapevi-SP: A. Araújo Silva Farmacêutica; 2009.

2. Hossain $P$, Kawar B, El Nahas M, Obesity and Diabetes in the Developing World - A Growing Challenge. N Engl J Med [Internet]. 2007 [cited 2012 Apr 20]; 356:213-5. Available from: http://www.nejm.org/doi/full/10.1056/NEJMp06817 3. Grant JF, Hicks N, Taylor AW, Chittleborough CR, Phillips PJ. The North West Adelaide Health Study Team. Gender-specific epidemiology of diabetes: a representative cross-sectional study. International journal for equity in health. [Internet]. 2009 [cited 2012 Apr 20]; 8:6. Available from:

http://www.equityhealthj.com/content/8/1/6

4. Dias JCR, Campos JADB. Diabetes mellitus: razão de prevalências nas diferentes regiões geográficas no Brasil, 2002 2007. Ciênc. saúde coletiva [Internet]. 2012 [cited 2012 Apr 20]; 17(1):239-44. Available from: http://www.scielo.br/scielo.php?script=sci_arttext\&pid=S1413$81232012000100026 \& \operatorname{lng}=e n \& n r m=i s o \&$ tlng=pt

5. Schmidt MI, Duncan BB, Hoffmann JF, Moura L, Malta DC, Carvalho RMSV. Prevalência de diabetes e hipertensão no Brasil baseada em inquérito de morbidade auto-referida, Brasil, 2006. Rev. saúde pública [Internet]. 2009 [cited 2012 Apr 19]; 43(suppl.2):7482. Available from:

http://www.scielo.br/scielo.php?script=sci_arttext\&pid=S0034$89102009000900010 \& \operatorname{lng}=$ en\&nrm=iso\&tlng=pt

6. Morais GFC, Soares MJGO, Costa MML, Santos IBC. O diabético diante do tratamento, fatores de risco e complicações crônicas. Rev. enferm. UERJ, Rio de Janeiro. 2009; 17(2):240-5.

7. Moreira LLR, Moreira MF, Nunes AB. Caracterização clínicoterapêutica de idosos diabéticos tipo 2 atendidos em hospital universitário. Rev Soc Bras Clin Med. 2009; 7:228-32.

8. Faustino EB, Azevedo EB, Silva PMC, Sales SS, Barros WPS. Diabetes Mellitus: busca ativa em portadores de obesidade. Cogitare enferm. 2011; 16(1):110-5.

9. Grossi SAA. O manejo do Diabetes Mellitus sob a perspectiva da mudança comportamental. In: Grossi SAA, Pascali PM (Orgs) Cuidados de enfermagem em Diabetes Mellitus. São Paulo: Sociedade Brasileira de Diabetes/Departamento de Enfermagem da Sociedade Brasileira de Diabetes; 2009. p 18-31.

10. Canavan RJMB. Diabetes and nondiabetes related lower extremity amputation incidence before and after the introduction of better organized diabetes foot care: contínuos longitudinal monitoring using a standart mehod. Diabetes Care. 2008; 31(3):45963.

11. Barreto SM, Passos VMAP, Giatti L. Comportamento saudável entre adultos jovens no Brasil. Rev Saúde Pública. 2009; 43(Supl 2):917. promoção à saúde de modo a detectar, precocemente, o DM2 na comunidade usuária dos serviços da Clínica de Enfermagem da UEPB, além de desenvolver medidas preventivas dentro da própria unidade de formação. $E$, ao mesmo tempo, os resultados fornecem subsídios para que os gestores implementem programas educativos relacionados à promoção da saúde e prevenção de doenças crônico-degenerativas, em particular, o DM2. Sugere-se, ainda, a realização de outros estudos com populações diferentes, buscando identificar mais associações e FR, como, por exemplo, quanto aos hábitos alimentares e aos níveis de colesterol.

12. Moreira TMM, Gomes EB, Santos JC. Fatores de risco cardiovasculares em adultos jovens com hipertensão arterial e/ou diabetes mellitus. Rev. Gaúcha Enferm. 2010; 31(4):662-9. 13. Bittencourt RJ, Chaves SR, Amado RC, Mendonça VF, Oliveira FJF, Antunes CMF. Validação de inquérito de risco referido para vigilância em saúde de fatores de risco de doença arterial coronariana em servidores públicos estaduais de Juiz de Fora, Minas Gerais, Brasil. Cad. Saúde Pública. 2004; 20(3):761-70.

14. Santos ECB, Teixeira CRS, Zanetti ML, Santos MA. A efetivação dos direitos dos usuários de saúde com diabetes mellitus: coresponsabilidades entre poder público, profissionais de saúde e usuários. Texto \& contexto enferm. 2011 Set; 20(3):461-70. 15. Field A. Descobrindo a Estatística usando o SPSS. 2a ed. Porto Alegre: Artmed; 2009.

16. Moraes SA, Freitas ICM, Gimeno SGA, Mondini L. Prevalência de diabetes mellitus e identificação de fatores associados em adultos residentes em área urbana de Ribeirão Preto, São Paulo, Brasil, 2006: Projeto OBEDIARP. Cad. saúde pública. 2010; 26(5):929-41. 17. Almeida VCF, Zanetti ML, Almeida PC, Damasceno MMC. Ocupação e fatores de risco para diabetes tipo 2: estudo com trabalhadores de enfermagem. Rev latinoam enferm. 2011; 19(3):476-84.

18. Sociedade Brasileira de Cardiologia. Sociedade Brasileira de Hipertensão. Sociedade Brasileira de Nefrologia. [VI Brazilian Guidelines on Hypertension]. Arq bras cardiol. 2010; 95(1 supl.1):151.

19. Matos LN, Giorelli GV, Saado A, Dias CB. Prevalence of prediabetes in patients with metabolic risk. Sao Paulo med. j. [Internet]. 2011 [cited 2012 Apr 22]; 129(5):300-8. Available from: http://www.scielo.br/scielo.php?pid=S1516$31802011000500004 \&$ script $=$ sci arttext

20. Lyra R, Silva RS, Montenegro Jr. RM, Matos MVC, Cézar NJB, Maurício-da-Silva L. Prevalência de diabetes melito e fatores associados em população urbana adulta de baixa escolaridade e renda do sertão nordestino brasileiro. Arq Bras Endocrinol Metab. 2010; 54(6): 560-6.

21. Kramer CK, Leitão CB, Pinto LC, Bolson P, Boza J, Silveiro SP, Gross JL, Canani LH. Risk factors for micro and macrovascular disease in black and white patients with type 2 Diabetes mellitus. Rev. Assoc. Med. Bras. 2009; 55(3):308-14.

22. Vilarinho RMF, Lisboa MTL, Thiré PK, França PV. Prevalência de fatores de risco de natureza modificável para a ocorrência de diabetes mellitus tipo 2. Esc. Anna Nery. 2008; 12(3):452-6. 23. Pinto LC, Ricardo ED, Leitão CB, Kramer CK, Zanatta CM, Gross $\mathrm{JL}$, Canani LH. Controle inadequado da pressão arterial em pacientes com diabete melito tipo 2. Arq. Bras. Cardiol. [Internet]. 2010 [cited 2012 Apr 20]; 94(5):651-5. Available from: http://www.scielo.br/scielo.php?script=sci_arttext\&pid=S0066782X2010000500013\&lng=pt\&nrm=iso

24. Knuth AG, Bielemann RM, Silva SG, Borges TT, Del Duca GF, Kremer MM, et al. Conhecimento de adultos sobre o papel da 
atividade física na prevenção e tratamento de diabetes e

hipertensão: estudo de base populacional no Sul do Brasil. Cad.

Saúde Pública. 2009; 25(3):513-20.

25. Lucas LPP, Barichello E, Zuffi FB, Barbosa MH. A percepção dos

portadores de Diabetes Mellitus tipo 2 em relação à amputação.

Rev. Eletr. Enf. [Internet]. 2010 [cited 2012 Apr 20]; 12(3):535-8.

Available from:

http://www.fen.ufg.br/revista/v12/n3/v12n3a17.htm.

Artigo recebido em 20/05/2011.

Aprovado para publicação em 14/04/2012.

Artigo publicado em 30/09/2012. 